p. 1333: in formula (3.7), the coefficient of $k_{1}^{2}$ is $m_{1}\left(m_{1}+m_{3}\right)$; in the formula for $\Delta$ the term of place 3,3 is $a_{1}^{2}-m_{3} a_{2}$

p. 1335: in the r.h.s. of the inequality, $\varepsilon^{2}$ should read $\varepsilon^{3}$

p. 1336: The integral in the last formula is

$$
\int_{R^{3}} \frac{\cos (x+y+z)-\cos (x+y)-\cos (x+z)-\cos (y+z)+\cos x+\cos y+\cos z-1}{x y z(x+y+z)} d x d y d z
$$

\title{
The Reunions of Three Dissimilar Vicious Walkers ${ }^{2}$
}

\section{E. Fisher and M. P. Gelfand}

On page 185, equation (3.4) should read, correcting subscripts in the two factors in parentheses,

$$
C_{3}\left(b_{1} \rightarrow 0, b_{2}=b_{3}\right)=x_{12} x_{13} x_{12,0} x_{13,0}\left(x_{13}^{2}-x_{12}^{2}\right)\left(x_{13,0}^{2}-x_{12,0}^{2}\right) / 3 \pi b^{8}
$$

In the first line of the following paragraph, the limit $b_{3} \rightarrow 0$ should be replaced by $b_{2} \rightarrow 0$.

${ }^{2}$ This paper appeared in J. Stat. Phys. 53:175 (1988). 